\title{
Plan d'action fédéral sur la résistance et le recours aux antimicrobiens au Canada.
}

\author{
Agence de la santé publique du Canada* en collaboration avec l'Agence canadienne d'inspection des \\ aliments, les Instituts de recherche en santé du Canada, Santé Canada, Agriculture et Agroalimentaire \\ Canada, Industrie Canada et le Conseil national de recherches Canada
}

\section{*Correspondance : Lindsay.noad@phac-aspc.gc.ca}

\section{Résumé}

En octobre 2014, le gouvernement du Canada a publié le document Résistance aux antimicrobiens et utilisation de ces derniers au Canada : un cadre d'action fédéral, suivi récemment du Plan d'action fédéral sur la résistance et le recours aux antimicrobiens au Canada : Prolongement du cadre d'action fédéral. Le Plan d'action fédéral présente des produits livrables concrets à l'appui des trois domaines prioritaires définis dans le cadre fédéral. Les principaux éléments du travail qui sera entrepris par l'Agence de la santé publique du Canada comprennent : établir un plan détaillé pour le Système canadien de surveillance de la résistance aux antimicrobiens pour renforcer la coordination et l'intégration d'activités et des renseignements relatifs à la résistance aux antimicrobiens (RAM) et à l'utilisation d'antimicrobiens, réaliser une analyse pour déterminer les lacunes potentielles dans les pratiques de prévention et de contrôle des infections et s'inspirer des leçons tirées de la campagne de sensibilisation à la résistance aux antimicrobiens de novembre 2014 pour éclairer les activités futures en matière de sensibilisation et d'éducation. Le gouvernement du Canada demeure engagé dans la prise de mesures relativement à la résistance aux antimicrobiens et au recours aux antimicrobiens et continuera de déterminer de nouvelles activités qui permettront de lutter contre la propagation de la résistance aux antimicrobiens. Le Plan d'action fédéral est un document évolutif qui sera régulièrement mis à jour afin de tenir la population canadienne informée des activités et des progrès en cours dans la mise en œuvre du cadre fédéral.

\section{Introduction}

La résistance aux antimicrobiens (RAM) représente une importante menace de santé publique à l'échelle mondiale, laquelle va en s'accroissant. En réponse à cette menace, le gouvernement du Canada a publié, en octobre 2014, le document Résistance aux antimicrobiens et utilisation de ces derniers au Canada : un cadre d'action fédéral (1). Le cadre présente une approche fédérale conjointe et coordonnée destinée à répondre à la menace de la résistance aux antimicrobiens par l'intermédiaire de mesures prises dans trois domaines : surveillance, intendance et innovation. D'autre part, le cadre sert de véhicule de discussion entre les partenaires et les intervenants au sujet des mesures collectives pouvant être prises pour améliorer l'efficacité des mesures individuelles au sein des secteurs concernés par la résistance aux antimicrobiens.

\section{Le Plan d'action}

Faisant suite au cadre, le Plan d'action fédéral sur la résistance et le recours aux antimicrobiens au Canada : Prolongement du cadre d'action fédéral (2) a été publié en mars 2015. Alors que le cadre de travail relève certaines mesures concrètes que peut prendre le gouvernement du Canada pour réduire la menace et les répercussions de la RAM, le Plan d'action vise à présenter des produits livrables précis entrepris par les ministères et organismes fédéraux en vertu de chaque mesure de suivi, ainsi que la date d'achèvement prévue pour chaque produit livrable. Le Plan d'action est un document évolutif qui sera régulièrement mis à jour afin de continuer de refléter le travail qui est en cours, et nos plans pour l'avenir.

\section{Une responsabilité partagée}

Comme l'indique le cadre, lutter contre la menace croissante que représente la résistance aux antimicrobiens au Canada constitue une responsabilité partagée dans tous les ordres du gouvernement (fédéral, provincial et territorial) et de multiples secteurs (p. ex. santé publique, soins de santé, santé des animaux, agriculture). 
Lorsque le cadre a été diffusé, il présentait le rôle fédéral dans la lutte contre la RAM de plusieurs ministères : Agence de la santé publique du Canada (ASPC), Santé Canada (SC), Agence canadienne d'inspection des aliments (ACIA), Instituts de recherche en santé du Canada (IRSC) et Agriculture et Agroalimentaire Canada (AAC). La portée du travail s'inscrivant dans le Plan d'action a été élargie pour inclure deux ministères fédéraux supplémentaires : Industrie Canada (IC) et le Conseil national de recherches du Canada (CNR).

Comme l'indique le cadre, les provinces, les territoires et les autres intervenants jouent aussi un rôle clé en vertu de leur responsabilité quant à la prestation des soins de santé, l'approbation des antimicrobiens pour la couverture médicale et la réglementation de l'utilisation des antimicrobiens dans la médecine vétérinaire et l'agriculture. Le plan d'action réaffirme l'engagement du gouvernement du Canada à collaborer avec l'ensemble des compétences territoriales et des intervenants pour concrétiser du plan d'action.

\section{Leadership}

Outre son travail dans les domaines de la surveillance, de l'intendance et de l'innovation, le gouvernement du Canada s'engage à endosser un rôle de leadership tant à l'échelle nationale qu'à l'échelle internationale. À l'appui de cet engagement, le Plan d'action met en avant les activités de leadership clé qui sont entreprises. Elles incluent : la collaboration du Canada avec des partenaires internationaux concernant l'élaboration et la mise en œuvre du Plan d'Action mondial de l'Organisation mondiale de la Santé sur la résistance aux antimicrobiens; la collaboration avec des partenaires et intervenants fédéraux, provinciaux et territoriaux et des intervenants de l'agroalimentaire et de l'industrie en santé humaine et animale pour élaborer un cadre pancanadien visant la résistance aux antimicrobiens; la coordination internationale des efforts de recherche et, dans le cadre du travail réalisé selon le Programme de sécurité sanitaire mondiale, l'appui apporté à l'élaboration d'une approche intégrée et globale des activités visant à lutter contre la résistance aux antimicrobiens chez les humains, les animaux et dans les secteurs agricole, alimentaire et environnemental.

\section{Surveillance}

\section{Mesure 1 : Établir et renforcer des systèmes de surveillance en vue de déterminer les nouvelles menaces ou l'évolution des tendances liées à la résistance aux antimicrobiens et à l'utilisation de ces derniers chez les humains et les animaux.}

Des renseignements exhaustifs sont nécessaires sur la portée du problème de résistance aux antimicrobiens, surtout hors des milieux hospitaliers ( $p$. ex. le mode d'utilisation des antimicrobiens, le nombre de bactéries résistantes et à quels médicaments, leur mode de propagation). Cette information est nécessaire pour déterminer la pleine ampleur du problème et elle est essentielle pour surveiller l'efficacité des interventions d'intendance; de plus, elle ne peut être recueillie que grâce à une surveillance efficace.

Au Canada, il existe déjà de bons systèmes de surveillance et nous poursuivrons ces activités de surveillance existantes, ainsi que les services de laboratoire appuyant les efforts destinés à combattre la résistance aux antimicrobiens. L'amélioration de notre surveillance de la résistance aux antimicrobiens s'appuiera également sur ces systèmes. Dans le cadre de ce domaine d'action, des exemples de certains produits livrables comprennent ce qui suit :

- $\quad$ L'Agence de la santé publique du Canada mettra en place un plan détaillé pour le Système national de surveillance de l'antimicrobiorésistance (SNSAR) en vue de renforcer la coordination et l'intégration des activités et des renseignements sur la résistance et le recours aux antimicrobiens. En outre, l'Agence procédera à un élargissement à une approche nationale par l'entremise de discussions avec des partenaires fédéraux, provinciaux et territoriaux et d'autres partenaires externes. (Date d'achèvement prévue : automne 2016)

- L'Agence canadienne d'inspection des aliments et Agriculture et Agroalimentaire Canada travailleront à l'élaboration des éléments clés d'un système de déclaration, de surveillance et de suivi concernant l'utilisation des antimicrobiens (UAM) dans le secteur agroalimentaire. Cela permettra de définir les besoins en matière de collecte de données et de déclaration parmi les intervenants et les décideurs, notamment les 
besoins en matière de déclaration à l'échelle internationale, nationale, provinciale et territoriale. (Date d'achèvement prévue : printemps 2016)

- Les Instituts de recherche en santé du Canada, ainsi que l'Agence de la santé publique du Canada, soutiendront les recherches et la diffusion des résultats concernant les études sur les interventions et la surveillance de la résistance aux antimicrobiens. (Date d'achèvement prévue : décembre 2019)

\section{Gestion}

La résistance aux antimicrobiens (RAM) a lieu lorsque des agents antimicrobiens sont utilisés; elle constitue donc un facteur constant dans les médecines humaine et vétérinaire. Ainsi, l'application de solides pratiques de gestion par tous est essentielle pour continuer à lutter contre la résistance aux antimicrobiens. Étant donné l'importance de la gestion, un certain nombre d'activités sont présentées dans le cadre des deux mesures dans ce domaine.

\section{Mesure 2 : Renforcer la promotion de l'utilisation appropriée des antimicrobiens en médecine humaine et} vétérinaire.

Un facteur important à prendre en compte dans le maintien de l'efficacité des médicaments antimicrobiens est la promotion d'efforts de prévention de la maladie en vue de minimiser ou d'éliminer le besoin en agents antimicrobiens. En plus de la prévention et du contrôle des infections, le gouvernement du Canada poursuivra ses efforts visant à faciliter et à améliorer l'éducation et la sensibilisation sur quand et comment utiliser des agents antimicrobiens, de même que le choix des agents antimicrobiens adéquats pour le traitement de certaines maladies. En outre, d'importants progrès ont été faits dans le secteur agroalimentaire afin de promouvoir l'utilisation prudente d'agents antimicrobiens dans la production d'aliments d'origine animale. Les principales activités qui seront réalisées prochainement et touchant les deux secteurs comprennent ce qui suit :

- L'Agence de la santé publique du Canada s'inspirera des leçons retenues de la campagne de sensibilisation à la résistance aux antimicrobiens de novembre 2014 pour consolider les activités futures de sensibilisation et d'éducation du grand public et des professionnels de la santé qui travaillent en milieu communautaire, hospitalier et de soins de longue durée. (Date d'achèvement prévue : printemps 2015)

- $\quad$ L'Agence de la santé publique du Canada effectuera une analyse pour déterminer les lacunes potentielles dans les pratiques de prévention et de contrôle des infections. (Date d'achèvement prévue : printemps 2016)

- L'Agence de la santé publique du Canada, Santé Canada, l'Agence canadienne d'inspection des aliments et Agriculture et agroalimentaire Canada entreprendront une série de consultations pour recenser les pratiques actuelles liées à l'utilisation d'agents antimicrobiens, cerner les meilleures pratiques en matière d'utilisation d'agents antimicrobiens, et déterminer la façon de tirer le meilleur parti des possibilités d'éducation existantes. (Date d'achèvement prévue : printemps 2016)

- $\quad$ Santé Canada et l'Agence canadienne d'inspection des aliments travailleront avec des promoteurs de médicaments pour faciliter leurs soumissions de modifications d'étiquette en vue de retirer les allégations de stimulation de la croissance de médicaments antimicrobiens importants sur le plan médical et avec les références connexes dans le Recueil des notices sur les substances médicatrices. (Date d'achèvement prévue : automne 2016)

- Santé Canada, l'Agence canadienne d'inspection des aliments et Agriculture et agroalimentaire Canada tiendront des discussions multilatérales avec des partenaires fédéraux, provinciaux et territoriaux et des intervenants afin d'examiner et de peaufiner des propositions pour accroître la supervision vétérinaire des agents antimicrobiens vétérinaires destinés à la production d'aliments d'origine animale. (Date d'achèvement prévue : printemps et été 2015)

- Les Instituts de recherche en santé du Canada appuieront la recherche et la diffusion des résultats découlant des recherches sur les mesures de gestion. (Date d'achèvement prévue : décembre 2019) 
Mesure 3 : Travailler avec les partenaires du secteur de l'agriculture animale pour renforcer le cadre réglementaire sur la médecine vétérinaire et les aliments médicamentés, y compris faciliter l'accès à des solutions de rechange et encourager l'adoption de pratiques visant à réduire l'utilisation d'antimicrobiens

- $\quad$ Outre les efforts visant l'amélioration des mesures de prévention et de contrôle des infections, ainsi que la promotion de l'éducation et de la sensibilisation, le gouvernement du Canada joue un rôle important dans la réglementation des produits de santé animale au Canada, y compris l'importation, la délivrance des licences et la vente de médicaments vétérinaires, d'aliments médicamenteux et de vaccins. À l'appui de ce rôle, les activités suivantes seront entreprises :

- Santé Canada et l'Agence canadienne d'inspection des aliments continueront d'accorder la priorité à la modernisation des cadres réglementaires sur la médecine vétérinaire et les aliments médicamenteux. (Date d'achèvement prévue pour les initiatives associées à la résistance aux antimicrobiens : décembre 2016).

- L'Agriculture et agroalimentaire Canada soutiendra les recherches dirigées par l'industrie pour confirmer l'efficacité des solutions de rechange aux antibiotiques dans les aliments offerts sur le marché, le cas échéant. (Date d'achèvement prévue : été 2015)

- Santé Canada va mettre au point des mesures à l'égard de l'importation des médicaments vétérinaires pour « utilisation personnelle » et renforcer le contrôle sur l'importation d'ingrédients pharmaceutiques actifs vétérinaires. (Date d'achèvement prévue : 2017)

- Les Instituts de recherche en santé du Canada soutiendront la recherche à l'interface entre la santé humaine et la santé animale et l'environnement dans le cadre de l'initiative Environnements et Santé. (Date d'achèvement prévue : printemps 2020)

\section{Innovation}

Mesure 4 : Promouvoir l'innovation grâce au financement d'une recherche collaborative et d'efforts de développement liés à la résistance aux antimicrobiens, tant au niveau national qu'au niveau international.

Tel qu'il est décrit dans le cadre fédéral, le gouvernement du Canada soutient la recherche et l'innovation nationales en cours en matière de santé, tout en collaborant avec les partenaires internationaux pour contribuer aux efforts de recherche mondiaux en matière de résistance et de recours aux antimicrobiens, de thérapies novatrices et de solutions de rechange. Certaines des activités principales dans ce domaine comprendront ce qui suit :

- Au moyen du Plan d'action sur la recherche, l'innovation et de développement de vaccins, la priorité est mise sur les vaccins novateurs en cours d'élaboration pour combattre certaines des menaces les plus importantes que pose la résistance aux antimicrobiens. (Date d'achèvement prévue : 2015)

- $\quad$ Agriculture et Agroalimentaire Canada continuera de soutenir et d'entreprendre des recherches et des mesures innovantes dans le domaine des améliorations des pratiques d'élevage d'animaux, de la prévention et du traitement des maladies, ainsi que de l'élaboration des vaccins, ce qui contribuera à la réduction des exigences relatives aux antimicrobiens dans l'industrie de l'élevage d'animaux. (En cours)

- Les Instituts de recherche en santé du Canada continueront de renforcer les connaissances pour créer des outils novateurs et des thérapies parallèles pour prévenir et limiter la propagation de la résistance aux antimicrobiens au moyen de la recherche et du développement par la concurrence ouverte et le financement stratégique par l'entremise de partenariats, et tout particulièrement collaborer avec des groupes internationaux pour favoriser les contributions du Canada et en tirer parti au sein d'équipes de recherche internationales : (En cours) 


\section{À l'avenir}

Le gouvernement du Canada demeure engagé dans la prise de mesures relativement à la résistance aux antimicrobiens et au recours aux antimicrobiens. À ce titre, nous continuerons de travailler avec les partenaires fédéraux, provinciaux, territoriaux et internationaux dans les domaines de la santé et de l'agriculture, ainsi qu'avec d'autres intervenants, pour déterminer la manière dont nous pouvons renforcer les activités existantes et prévues. Nous continuerons aussi de travailler en vue de déterminer de nouvelles activités qui permettront de lutter contre la propagation de la résistance aux antimicrobiens. Le plan d'action fédéral est le premier d'une série pour tenir la population canadienne informée de nos activités et de nos progrès en cours dans la mise en œuvre du cadre fédéral.

\section{Remerciements}

Mille mercis à tous les collaborateurs à l'Agence de la santé publique du Canada, Santé Canada, l'Agence canadienne d'inspection des aliments, les Instituts de recherche en santé du Canada, Agriculture et Agroalimentaire Canada, Industrie Canada et le Conseil national de recherches du Canada.

\section{Conflit d'intérêts}

\section{Aucun}

\section{Références}

(1) Public Health Agency of Canada. Antimicrobial Resistance and Use in Canada: A Federal Framework for Action. 2014 OCT 24. http://healthycanadians.gc.ca/drugs-products-medicaments-produits/antibiotic-resistanceantibiotique/antimicrobial-framework-cadre-antimicrobiens-eng.php.

Disponible en français : http://healthycanadians.gc.ca/drugs-products-medicaments-produits/antibiotic-resistanceantibiotique/antimicrobial-framework-cadre-antimicrobiens-fra.php.

(2) Public Health Agency of Canada. Federal Action Plan on Antimicrobial Resistance and Use in Canada: Building on the Federal Framework for Action. 2015 Mar 31. http://healthycanadians.gc.ca/publications/drugs-productsmedicaments-produits/antibiotic-resistance-antibiotique/action-plan-daction-eng.php. Disponible en français : http://healthycanadians.gc.ca/publications/drugs-products-medicaments-produits/antibioticresistance-antibiotique/action-plan-daction-fra.php. 